\title{
ICA and Neural Networks for Kannada Signature Identification
}

Rajesh T.M ${ }^{1}$, V.N. Manjunath Aradhya ${ }^{2}$

\begin{abstract}
In biometrics, signature is one of the largest and oldest part used for endorsement. In this paper, approach based on Independent Component Analysis (ICA) and neural networks is proposed to study on Kannada Signatures. Experiment on 5000 samples of Kannada signature has been considered and the result is found to be accurate and promising.

Key words: Kannada Signature Identification, Independent Component Analysis(ICA), NN.
\end{abstract}

\section{INTRODUCTION}

The signature recognition is an active and challenging research area due to its complexities like intra class variations. The signature recognition can be categorized into online signature recognition and offline signature recognition. The main steps of signature recognition system are preprocessing, thinning, feature extraction and recognition. The feature used in offline signature images are, geometric centers, angle and distance of a pixel from a reference point, signature height and width. Offline recognition of signature is done by regarding an image of the signature which is getting by using scanner and extracting its features. Offline signature recognition is difficult because of limited amount of features which can be extracted.

\section{LiteratURE SURVEY}

Comparative analysis of offline signature verification system is presented by Yadav and Tyagi [1]. The work concentrates on digital signature which has been used for authentication of the user for their validity. McCabe et al. [2] has described a handwritten signature verification based on neural network method. They have extracted various static and dynamic signature features to train the NN architecture. Different types of network topologies have tested and also, the comparison study has been done. The experimentation study concluded that, the performance of the system is $3.3 \%$ error rate.

Offline handwritten signature verification using local and global feature is introduced by Pham et

${ }^{1}$ Computer Science \& Engineering Department, Jain University Bangalore, Karnataka, India

${ }^{2}$ Dept of MCA, Sri Jayachamaraja College of Engineering, Mysore University Mysore, Karnataka, India 
al. [3]. The method presented an offline signature verification and exploits both the intensity and geometry features. In experimentation they have been used standard datasets of Sig comp 2011.

Haque and Ali [5] used parallel block analysis for offline signature verification. A signature image is separated into blocks and then each block is comprised by a set of features. In experimentation they have been used database of 1225 signature images and also they have collected twenty-four forgery signature of each person from the randomly selected groups. In experimentation, fifteen original signatures and twenty-four forgery signatures are used to testing purpose.

The supervised neural network approach is employed on offline handwritten signature verification system by Jarad et al. [6]. Recognition and verification of handwritten signature used an artificial neural network based on Back propagation algorithm. In experimentation they have been used 400 signatures, which includes original and forged signature of twenty individual persons. Odeh and Khalil [7], used neural network approach on offline signature verification and recognition. The method applies MLP neural network technique for offline signature verification and recognition. This technique consists of four different features like, eccentricity, skewness, kurtosis, and orientation. Finally, the authenticity of the signatures is verified.

An artificial neural network has been used for evaluation on offline signature verification by khalifa et al. [8]. The method discusses a nonlinear approach for offline signature verification. Hybrid writer dependent and writer independent offline signature verification system is introduced by Eskander et al. [9]. The method WI -classification is used to verify his queries. During the operation, user samples are collected and adapt the WI classifier to his signature databases. Offline signature verification system using grid and tree based feature extraction is described by Shukla et al. [10]. The method verifies the handwritten signature of a particular person and also they have matched each person signatures with the other signatures.

A review of handwritten signature verification systems and methodologies is described by Padmanjali and Aprameya [11]. A review of research work and methodologies in the field of handwritten signature verification and various classifiers and matching techniques used lists in signature verification. Fractal approach is used for Identification and verification of offline handwritten signature by Zouari et al. [12]. The method mainly focused on identification and verification of offline handwritten signatures. In experimentation they have used FUM-PHSDB database. The method depicted an identification rate of $95 \%$ accuracy and a verification rate of $83 \%$ accuracy.

\section{Proposed Methodology}

The aim of ICA is to remove the correlations exist in data. Based on non-Gaussianity, independent component analysis (ICA) separates the components, but for perfect Gaussianity sources cannot be separated. The ICA optimizes the independence condition by minimizing the 
correlation between components and these components are representing the different nonoverlapping information. ICA obtains the independent sub components by separating the statistically dependent mixture components. This section influences how to make use of the selected independent component representation for recognition.

ICA for handwritten signature will be operating on two different types of architectures they are Architecture I or Architecture II. In Architecture I, the mentioned signature images have been considered as a linear mixture of statistically independent basis. ICA constructs uses of the reconstructive coefficients of a signature image are formed from these ground images. These coefficient members of all images might be reciprocally dependent but the ground images are mutually independent. In Architecture II, nevertheless, ICA is applied to find a set of statistically independent coefficients to constitute an image and the resulting ground images may be reciprocally dependent. In the subsequent sections, we are going to analyze two architectures of independent component analysis and their corresponding principal component analysis baseline algorithms.

\section{A. ICA Architecture I}

Initially we have given a set of $\mathrm{M}$ training samples $a_{1}, a_{2}, a_{3} \ldots \ldots . . a_{M}$ in $\square^{N}$ we organize the image column data matrix $A=\left(a_{1}, a_{2}, a_{3} \ldots \ldots \ldots a_{M}\right)$ and its, transpose $B=A^{T}$. In ICA Architecture I, the signature images have considered as stochastic variable and the same time picture element values are allowed for watching of these variables. This intends the independent component analysis is executed on the image row data matrix B. Rewriting $B=\left(b_{1}, b_{2}, b_{3} \ldots \ldots . . . b_{N}\right)$ its column vectors $b_{1}, b_{2}, b_{3} \ldots \ldots \ldots \ldots b_{N}$ has been used as observance to appraised the un mingled matrix of the ICA model.

\section{1) Focusing or Centering on Data}

Initially we are focusing on the data $\mathrm{B}$, which is an observation space $\square^{M}$ and also we are going to find out its mean vector, where $\mu_{I}=\left(\mu_{1}, \mu_{2}, \mu_{3}, \ldots \ldots . . . \mu_{M}\right)^{T}$. Actually, $\mu_{j}=E\left\{a_{j}\right\}$, that is the mean vector $\mu_{I}$ from each observation vector, that is, $\bar{b}_{j}=\left(b_{j}-\mu_{I}\right)$. After that we observed row data matrix of focusing images $B_{h}=\left(\bar{y}_{1}, \bar{y}_{2}, \bar{y}_{3}, \ldots \ldots \ldots . . . \bar{y}_{N}\right)$.

Let define $A_{v}=B_{h}^{T}=\left(\tilde{a}_{1}, \tilde{a}_{2}, \tilde{a}_{3}, \ldots \ldots \ldots \tilde{a}_{M}\right) . A_{v}$ is addressed that vertically centered image column data matrix and each column $\bar{A}_{j}$ is a zero-mean image, ie., mean of all pixel values have been removed out from the original images.

\section{2) Sphericity Data using PCA}

The data using PCA founded on the centered notices vectors $\bar{b}_{1}, \bar{b}_{2}, \bar{b}_{3} \ldots \ldots \ldots . \bar{b}_{N}$. The Co-variance matrix is given by 
$\sum_{I}=\frac{1}{N} \sum_{j=1}^{N} \bar{b}_{j} \bar{b}_{j}^{T}=\frac{1}{N} B_{h} B_{h}^{T}$

Let $C_{v}=B_{h} B_{h}^{T}$. Compute the orthogonal Eigen vectors $\gamma_{1}, \gamma_{2}, \gamma_{3} \ldots \ldots \ldots . \gamma_{m}$ of $C_{v}$. It is accompany to the $\mathrm{m}$ largest positive Eigen values $\lambda_{1} \geq \lambda_{2} \geq \lambda_{3} \geq \ldots \ldots \ldots . . . \lambda_{m}$. And then $\mathrm{m}$ largest positive Eigen values of $\sum_{I}$ are $\lambda_{1} / N, \lambda_{1} / N \ldots \ldots . . \lambda_{m} / N$ and the affiliated orthogonal eigenvectors are $\gamma_{1}, \gamma_{2}, \gamma_{3} \ldots \ldots$ and $\gamma_{m}$.

Let $V=\left(\gamma_{1}, \gamma_{2}, \gamma_{3} \ldots \ldots \ldots . \gamma_{m}\right), \Lambda=\operatorname{diag}\left(\lambda_{1}, \lambda_{2}, \lambda_{3} \ldots \ldots . \lambda_{m}\right)$,

We find the matrix $D=[(1 / N) \Lambda]^{-1 / 2}=\sqrt{N} V \Lambda^{-1 / 2}$, Such that

$D^{T}=\sum_{I} D=I$

The data matrix $B_{h=}\left(\bar{b}_{1}, \bar{b}_{2}, \bar{b}_{3} \ldots \ldots . . . \bar{b}_{N}\right)$ can be using the translate.

$$
E=D^{T} B_{h}
$$

Let us reconstructing the following Co-variance matrix founded on the vertically centered image column data matrix $A_{v}=B_{h}^{T}=\left(\tilde{a}_{1} \tilde{a}_{2}, \tilde{a}_{3,}, \ldots \ldots . . \tilde{a}_{M}\right)$ :

$\sum_{v}=\frac{1}{M} A_{v} A v_{v}^{T}=\frac{1}{M} B B_{h} B v_{h}^{T}$

\section{3) ICA process}

In this step we are going to execute ICA on E, after that we will get the matrix $F_{I}$ with $\mathrm{m}$ independent ground images in its row, that is,

$U_{I}=G_{I} E$

Where, $G_{I}$ is combined matrix return by an inclined ICA algorithm founded on the input data E. Choosing notice that the combine matrix should be multiplicative inverse from the eq. (5), it described that

$$
E=G_{I}^{-1} F_{I}
$$

Afterwards vertically focusing and projecting an inclined image ' $a$ ' in a column vector onto the row vectors of $\mathrm{E}$. We have $h=E a$; ' $\mathrm{E}$ ' is a principal eigenvector of $\Sigma_{V}$ a minimum-square-error representation of ' $a$ ' is

$\hat{a}=E^{T} h$

Replacing En.(6) into En.(7), we have

$\hat{a}=F_{I}^{T}\left(G_{I}^{-1}\right)^{T} h$

Hence, in the distance traversed by the row vectors of $F_{I}$ and the vector of an internal representation co-efficient of images $\mathrm{A}$ is given by

$k=\left(G_{I}^{-1}\right)^{T} h=\left(G_{I}^{-1}\right)^{T} E a$

Such translation can also be into the following two types of transformation.

$h=E a$

$k=\left(G_{I}^{-1}\right)^{T} h$

Because the row vectors of $E$ are principal diagonal vectors of $\Sigma_{V}$, the translation in Eq.(10) is a 
exceptional PCA transform, here each data is focused on vertically. This translation is therefore called as vertically focused on PCA. Since the ICA architecture I requires a vertically focused on PCA process. Incidental, if the combine matrix $M$ is orthogonal, the equation $G_{I}{ }^{-1}=G_{I}{ }^{T}$ applies; and then Eq. (9) becomes,

$k=G_{I} E a=F_{I} a$

In this instance, we have seen that $k$ is prevailed by directly projecting the sample a onto $\mathrm{m}$ independent ground.

\section{B. ICA Architecture II}

In this architecture, we have given a set of $M$ training samples $a_{1}, a_{2}, a_{3} \ldots \ldots . . . a_{M} \operatorname{in} \square^{N}$, we sort the image column data matrix $A=\left(a_{1}, a_{2}, a_{3} \ldots \ldots \ldots a_{M}\right)$. The input data images are used in architecture II is determined by using statistically independent coefficients. In this architecture, the handwritten signature images are considered as observation, and the pixel's values are random variables. ICA has executed directly on the image column data matrix A.

\section{1) Focusing or Centering on Data}

Let us focus on the observation vector space $\square^{n}$. The mean vector $\mu_{I I}=E\{a\}=(1 / M) \sum_{j=1}^{M} a_{j}$, here all observation vectors are subtracted by the mean vector $\mu_{I I}$, after that we are going to focusing on column data matrix $A_{h}=\left(a_{1}, a_{2}, a_{3} \ldots \ldots \ldots a_{M}\right) . A_{h}$ is called as the horizontally focused image column data matrix.

\section{2) Sphericity Data using PCA}

Established on the horizontally focused image vectors $a_{1}, a_{2}, a_{3} \ldots \ldots . . . a_{M}$, we can reconstruct the following co-variance matrix.

$\sum_{I I}=\frac{1}{M} \sum_{j=1}^{M} \bar{a}_{j} \bar{a}_{j}^{T}=\frac{1}{M} A_{h} A_{h}^{T}$

Suppose $\beta_{1}, \beta_{2}, \beta_{3} \ldots \ldots \ldots \ldots$ and $\beta_{m}$ are the orthogonal eigenvectors of $\sum_{I I}$ comparing to the $\mathrm{m}$ largest positive Eigen values $\lambda_{1} \geq \lambda_{2} \geq \lambda_{3} \geq \ldots \ldots \ldots . \lambda_{m}$. Allowing $D_{h}=\left(\beta_{1}, \beta_{2}, \beta_{3} \ldots \ldots \ldots . . . \beta_{m}\right)$, and the standard principal component analysis transform is $h=D_{h}^{T} a$

Let $D_{w}=D_{h} \Lambda^{-1 / 2}$, where $\Lambda=\operatorname{diag}\left(\lambda_{1}, \lambda_{2}, \lambda_{3} \ldots \ldots . \lambda_{m}\right)$, we have $D_{w}^{T} \sum_{I I} D_{w}=I$, the PCA translation is $H=D_{w}{ }^{T} a$

\section{3) ICA process}

We after that perform ICA, on the spherical data $h_{1}, h_{2}, h_{3}, \ldots \ldots \ldots \ldots . .$. and $h_{m}$. Imagine the resulting combine matrix is $G_{I I}$. The whole transform matrix $F_{I I}$ of ICA architecture II is 


$$
F_{I I}=E_{I I} D_{w}^{T}
$$

For a established image a in a column vector, after all horizontally focused combine by $F_{I I}$, we have

$$
k=F_{I I} a=G_{I I} D_{w}{ }^{T} a
$$

In a recognition purpose the vector $k$ holding a set of independent coefficients is used to comprise the image. It is apparent that the transformation in (Eq.17) can be disintegrated in to two items. PCA transform $h=D_{w}^{T} a$ and complete ICA projection $k=G_{I I} h$. Afterwards the ICA architecture II needs not only a horizontal focused on PCA but also focused on whitened PCA. In an Architecture II, the principal component analysis process has taken for evaluating independent component analysis.

Many of the classifiers which use techniques to learn are designed in such a way that it is suitable to implement in digital computers. However, the neural networks have been designed in such a way that mimic the way in which human brains learns. In this work the use of different NN architecture such as, generalized regression neural network (GRNN), probabilistic neural network (PNN) architectures are used. More detail description regarding NN architectures can be seen in [13].

\section{EXPERIMENTAL RESULTS}

In order to evaluate the performance of the proposed system, we have created our own dataset. This dataset consists of 100 individual Kannada signatures and each consists of 50 samples. Figure 1 shows the sample Kannada signatures.

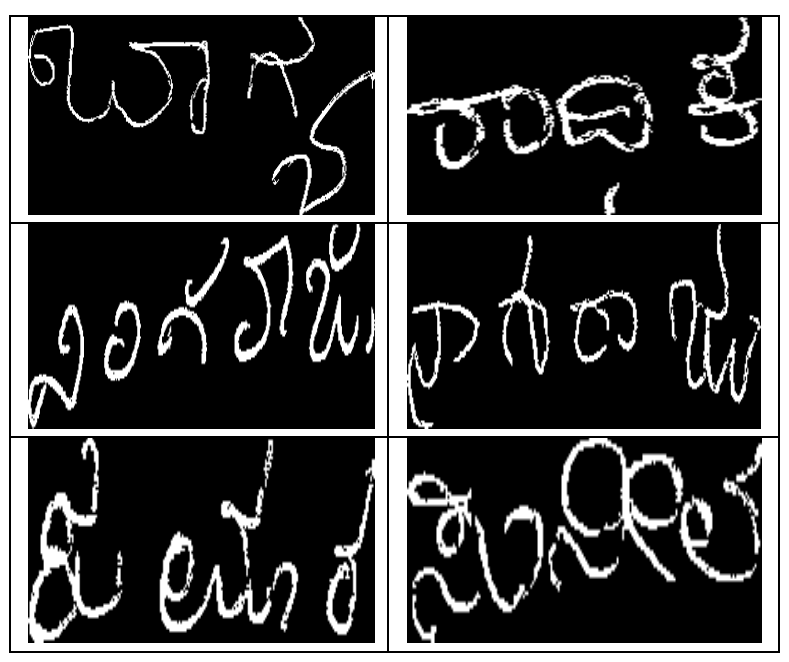




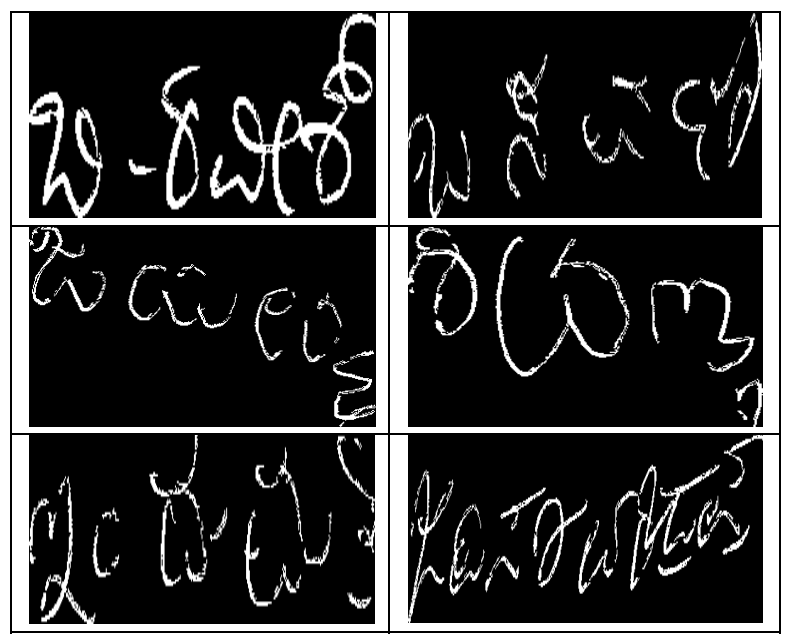

Figure 1: Some of the sample images of Kannada signatures.

Table 1 shows the performance of the proposed method on the dataset considered. We have divided the dataset into training and testing. Three types of experiment are considered. In the first type, 20 samples are used for training and remaining samples are used for testing purpose. In the second type and third type, 30 and 40 samples are considered for training and remaining samples are used for testing purpose respectively. From the table it is quite evident that ICA I performs better compared to architecture II.

Table 1: Recognition accuracy

\begin{tabular}{|l|l|l|l|}
\hline $\begin{array}{c}\text { Training: } \\
\text { Testing / } \\
\text { Methods }\end{array}$ & $\mathbf{2 0 : 3 0}$ & $\mathbf{3 0 : 2 0}$ & $\mathbf{4 0 : 1 0}$ \\
\hline ICA I & 83.8 & $\mathbf{8 8 . 6}$ & 89.8 \\
\hline ICA II & 81.5 & 85.3 & $\mathbf{9 0 . 0}$ \\
\hline ICA I GRNN & 84.3 & 87.0 & $\mathbf{9 0 . 1}$ \\
\hline ICA I - PNN & 84.3 & 87.0 & $\mathbf{9 0 . 3}$ \\
\hline $\begin{array}{l}\text { ICA II - } \\
\text { GRNN }\end{array}$ & 83.6 & 86.5 & $\mathbf{9 0 . 1}$ \\
\hline
\end{tabular}

\section{Conclusion}

The Independent component analysis has been discussed and used to recognize the signature. ICA optimizes the independence condition by minimizing the correlation between components. Here, both ICA architecture I and architecture II have used to extract the features of signatures. The performance of both architectures are evaluated on the own dataset and the recognition rate is evaluated. In future work, the work will be extended by different classifiers to recognize the signatures of south India. 


\section{REFERENCES}

[1] Yadav, Deepti, and Ranbeer Tyagi. "Comparative Analysis of Offline Signature Verification System." International Journal of Signal Processing, Image Processing and Pattern Recognition 8.11 (2015): 355-364.

[2] McCabe, Alan, Jarrod Trevathan, and Wayne Read. "Neural network-based handwritten signature verification." Journal of computers 3.8 (2008): 9-22.

[3] Le, Hong-Ha, and Nang-Toan Do. "Offline handwritten signature verification using local and global features." Annals of Mathematics and Artificial Intelligence 75.1-2 (2015): 231-247.

[4] P. Shankar Rao, J. Aditya "Handwriting Recognition - "Offline" Approach".

[5] Haque, Md Asraful, and Tofik Ali. "Improved offline signature verification method using parallel block analysis." Recent Advances in Computing and Software Systems (RACSS), 2012 International Conference on. IEEE, 2012.

[6] Jarad, Mujahed, Nijad Al-Najdawi, and Sara Tedmori. "Offline handwritten signature verification system using a supervised neural network approach."Computer Science and Information Technology (CSIT), 2014 6th International Conference on. IEEE, 2014.

[7] Kruthi, C., and Deepika C. Shet. "Offline signature verification using support vector machine." Signal and Image Processing (ICSIP), 2014 Fifth International Conference on. IEEE, 2014.

[8] Alam, Md Khorshed, and Aisha Hassan Abdalla. "An evaluation on offline signature verification using artificial neural network approach." Computing, Electrical and Electronics Engineering (ICCEEE), 2013 International Conference on. IEEE, 2013.

[9] Eskander, George S., Robert Sabourin, and Eric Granger. "Hybrid writer-independentwriter-dependent offline signature verification system." IET biometrics 2.4 (2013): 169-181.

[10]Shukla, Amit Kishore, et al. "Offline signature verification system using grid and tree based feature extraction." Issues and Challenges in Intelligent Computing Techniques (ICICT), 2014 International Conference on. IEEE, 2014.

[11]Padmajadevi, G., and K. S. Aprameya. "A review of Handwritten Signature Verification Systems and Methodologies."

[12]Zouari, Ramzi, Raouia Mokni, and Monji Kherallah. "Identification and verification system of offline handwritten signature using fractal approach."Image Processing, Applications and Systems Conference (IPAS), 2014 First International. IEEE, 2014.

[13]Manjunath Aradhya V N, "An Attempt towards the development of UOCR for the recognition of Multilingual documents", Ph.D. thesis, University of Mysore, India, 2007. 